\title{
ПРЕОБРАЗОВАНИЕ ОПТИЧЕСКИХ ВИХРЕЙ ПОЛЯРИЗАЦИОННЫМИ ДИНАМИЧЕСКИМИ ГОЛОГРАММАМИ
}

\author{
(C) 2013 г. О. Г. Романов, Д. В. Горбач, А. Л. Толстик \\ Белорусский государственный университет, 220030 Минск, Беларусь \\ E-mail: romanov@bsu.by,tolstik@bsu.by \\ Поступила в редакцию 22.01.2013 г.
}

\begin{abstract}
Представлены результаты теоретического и экспериментального исследований закономерностей преобразования топологической и поляризационной структур оптических вихрей поляризационными динамическими голограммами, сформированными импульсными гауссовыми и сингулярными световыми пучками в растворах красителей.
\end{abstract}

DOI: $10.7868 / \mathrm{S} 0030403413090201$

Как правило, при записи как статических, так и динамических голограмм используют одинаково поляризованные волны, когда пространственно модулированное интерференционное поле опорной и сигнальной волн приводит к записи голографических решеток за счет пространственной модуляции оптических параметров фоточувствительной среды (изменение показателя преломления и (или) коэффициента поглощения). При поляризационной голографической записи опорная и сигнальная волны поляризованы ортогонально друг другу, суммарная интенсивность этих волн остается постоянной, и имеет место только пространственная модуляция состояния поляризации света в соответствии с разностью фаз записывающих голограмму волн [1]. При этом пространственно-переменное состояние поляризации суммарного поля вызывает в среде возникновение пространственной модуляции фотоанизотропии и (или) фотогиротропии [2-4]. Переход к поляризационной записи голограмм позволяет управлять поляризацией дифрагированного излучения, что может быть использовано, например, в системах коррекции лазерного излучения со сложным распределением поляризации по фронту волны [5], а также получать информацию о строении и анизотропных свойствах среды, что имеет перспективы использования в системах поляризационной оптической памяти [6].

Поляризационная запись динамических голограмм в растворах сложных органических соединений (красителей) возможна вследствие наведенной анизотропии поглощения среды. При этом эффект насыщения поглощения приводит к проявлению нелинейностей пятого и более высоких порядков, определяющих возможность реализации нелинейной голографической записи и многоволновых процессов [7-9]. Многоволновые взаимодействия на основе нелинейных дина- мических голограмм, в свою очередь, позволяют реализовать управление в реальном времени структурой оптических вихрей, при этом становится возможным преобразование их волнового фронта [10], топологической структуры [11], а также частоты несущей волны [12].

В настоящей работе проведен теоретический анализ процессов преобразования топологической и поляризационной структур оптических вихрей в схеме записи и считывания пропускающих динамических поляризационных голограмм, а также приведены результаты экспериментальных исследований закономерностей преобразования сингулярных пучков при реализации поляризационного четырехволнового взаимодействия (ЧВB).

\section{ТЕОРЕТИЧЕСКАЯ МОДЕЛЬ}

Рассмотрим задачу воздействия высокоинтенсивного поляризованного лазерного излучения на резонансную среду, учитывающую два энергетических состояния (основное и возбужденное). Предположим, что световой пучок $\mathbf{E}$ распространяется вдоль оси $z$ и является линейно поляризованным вдоль оси $x$. Исходя из кинетических уравнений для населенностей основного $\left(n_{1}\right)$ и возбужденного $\left(n_{2}\right)$ энергетических уровней [13] можно получить стационарные функции распределения в единице телесного угла $\Omega$, зависящие от интенсивности излучения $I$ и угла $\theta$ между электрическим вектором Е и дипольным моментом молекул D:

$$
n_{1}(I, \Omega)=\frac{1}{8 \pi} \frac{2+3 \alpha I \cos ^{2} \theta}{1+3 \alpha I \cos ^{2} \theta}
$$




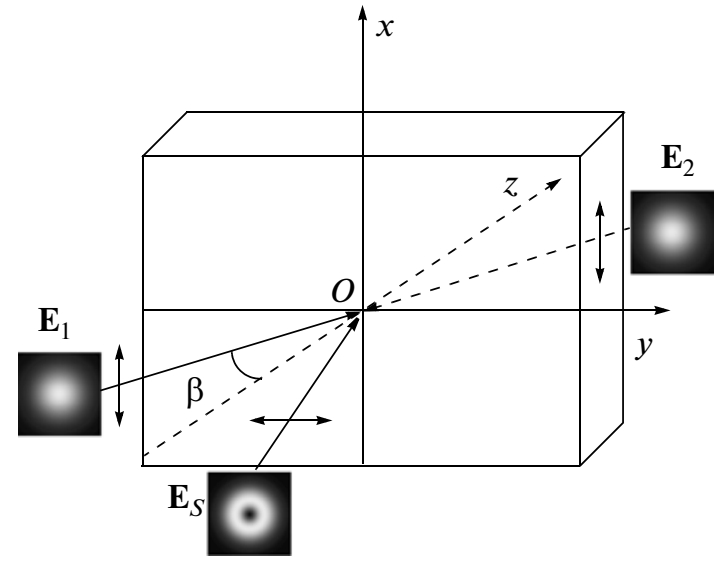

Рис. 1. Схема формирования объемной поляризационной голограммы опорным гауссовым и сигнальным сингулярным световыми пучками.

$$
n_{2}(I, \Omega)=\frac{1}{8 \pi} \frac{3 \alpha I \cos ^{2} \theta}{1+3 \alpha I \cos ^{2} \theta}
$$

где коэффициент $\alpha=\left(B_{12}+B_{21}\right) / v p_{21}$ определяет интенсивность насыщения резонансного перехода $\left(I_{\text {нас }}=\alpha^{-1}\right), B_{12,21}-$ коэффициенты Эйнштейна для вынужденных переходов, $P_{21}-$ суммарная вероятность спонтанных и безызлучательных переходов, $v=c / n_{0}-$ скорость света в среде с показателем преломления $n_{0}$.

Для описания нелинейного отклика ансамбля двухуровневых частиц используем формализм комплексного показателя преломления $\hat{n}=n+i \kappa$. Коэффициенты экстинкции среды вдоль оси поляризации $x$ и перпендикулярной ей оси $y$ определяются следующими выражениями:

$$
\kappa^{(x, y)}=\int \kappa^{(x, y)}(\Omega) d \Omega=\iint \kappa^{(x, y)}(\theta, \phi) \sin \theta d \theta d \phi,
$$

где функции

$$
\kappa^{(x, y)}(\theta, \phi)=(\hbar c N / 2 \vee)\left(n_{1}-n_{2}\right) b^{(x, y)}(\theta, \phi)
$$

определяют вклад в коэффициент экстинкции частиц, ориентированных в единичном телесном угле $\Omega$. При этом используются дифференциальные коэффициенты Эйнштейна для вынужденных переходов

$$
\begin{aligned}
& b^{(x)}(\theta, \phi)=3 B \sin ^{2} \theta \cos ^{2} \phi, \\
& b^{(y)}(\theta, \phi)=3 B \sin ^{2} \theta \sin ^{2} \phi,
\end{aligned}
$$

$B_{12}=B_{21} \equiv B$ для совпадающих контуров поглощения и люминесценции. Интегрируя (3) по всем углам $\theta$ и $\phi$, получаем следующие выражения для коэффициентов экстинкции среды вдоль оси поляризации $x$ и перпендикулярной ей оси $y$ :

$$
\begin{gathered}
\kappa^{(x)}=3 \kappa_{0}\left[\frac{1}{3 \alpha I}-\frac{\operatorname{arctg} \sqrt{3 \alpha I}}{(3 \alpha I)^{3 / 2}}\right] \\
\kappa^{(y)}=\frac{3}{2} \kappa_{0}\left[-\frac{1}{3 \alpha I}+\left(\frac{1}{\sqrt{3 \alpha I}}+\frac{1}{(3 \alpha I)^{3 / 2}}\right) \operatorname{arctg} \sqrt{3 \alpha I}\right]
\end{gathered}
$$

где $\kappa_{0}-$ не зависящий от интенсивности (линейный) коэффициент экстинкции.

Вычислим анизотропию коэффициента экстинкции $\left(\kappa_{x}-\kappa_{y}\right) / \kappa_{0}$ при малой интенсивности светового пучка $(\alpha I \ll 1)$, используя при этом разложение $\operatorname{arctg} \sqrt{3 \alpha I} \sim \sqrt{3 \alpha I}(1-\alpha I)$ :

$$
\left(\kappa_{x}-\kappa_{y}\right) / \kappa_{0}=3 \alpha I / 2 .
$$

Аналогично можно рассчитать анизотропию показателя преломления двухуровневой резонансной среды под действием интенсивного поляризованного излучения:

$$
\left(n_{x}-n_{y}\right) / \kappa_{0}=3 \Theta \alpha I / 2 B,
$$

где функция $\Theta(\omega)$ связана соотношением Крамерса-Кронига с коэффициентом Эйнштейна для вынужденных переходов $B(\omega)$. Таким образом, при интенсивности светового пучка, много меньшей интенсивности насыщения резонансного перехода, данная модель описывает эффекты светоиндуцированной анизотропии коэффициента поглощения и показателя преломления в приближении кубической нелинейности.

Рассмотренная модель взаимодействия высокоинтенсивного линейно поляризованного излучения с нелинейной первоначально изотропной средой была применена для описания процесса преобразования пространственной и топологической структур вихревых оптических пучков при записи и считывании поляризационных динамических голограмм. Будем полагать, что частота $\omega$ записывающих динамическую голограмму световых пучков $\mathbf{E}_{1}$ и $\mathbf{E}_{S}$, а также считывающего пучка $\mathbf{E}_{2}$ близка к центру полосы поглошения $S_{0}-S_{1}$ раствора красителя. Опорный пучок $\mathbf{E}_{1}$ с гауссовым распределением интенсивности в поперечном профиле и сигнальный сингулярный световой пучок $\mathbf{E}_{S}$ пересекаются в объеме нелинейной среды под небольшим углом $\beta$, а считывающий гауссов пучок $\mathbf{E}_{2}$ распространяется точно навстречу пучку $\mathbf{E}_{1}$ (рис. 1). 
Ограничим рассмотрение случаем линейной взаимно ортогональной поляризации опорного и сигнального пучков $\left(\mathbf{E}_{1}\left\|\mathbf{x}, \mathbf{E}_{s}\right\| \mathbf{y}\right)$, полагая при этом, что поляризация считывающего динамическую голограмму пучка совпадает с поляризацией опорного $\left(\mathbf{E}_{2} \| \mathbf{E}_{1}\right)$. В этом случае суммарное поле световых волн можно представить в виде

$$
\begin{aligned}
\mathbf{E}= & \mathbf{E}_{1}+\mathbf{E}_{S}=\frac{1}{2}\left[\mathbf{e}_{1} E_{1 x} \exp i\left(\mathbf{k}_{1} \mathbf{r}-\omega t+\varphi_{1}\right)+\right. \\
& \left.+\mathbf{e}_{2} E_{S y} \exp i\left(\mathbf{k}_{S} \mathbf{r}-\omega t+\varphi_{S}\right)+\text { K.c. }\right],
\end{aligned}
$$

где $\mathbf{e}_{1}, \mathbf{e}_{2}-$ единичные векторы вдоль осей $x$ и $y$ соответственно, $\mathbf{k}_{1}$ и $\mathbf{k}_{S}-$ волновые векторы опорной и сигнальной волн.

Преобразуем выражение (8) к виду

$$
\mathbf{E}=\left[\begin{array}{c}
E_{x} \\
E_{y} \\
E_{z}
\end{array}\right]=\left[\begin{array}{c}
p_{x} \cos \omega t+q_{x} \sin \omega t \\
p_{y} \cos \omega t+q_{y} \sin \omega t \\
0
\end{array}\right],
$$

где

$$
\begin{gathered}
p_{x}=E_{1 x} \cos \left(\mathbf{k}_{1} \mathbf{r}+\varphi_{1}\right), \quad q_{x}=E_{1 x} \sin \left(\mathbf{k}_{1} \mathbf{r}+\varphi_{1}\right), \\
p_{y}=E_{S y} \cos \left(\mathbf{k}_{S} \mathbf{r}+\varphi_{S}\right),
\end{gathered}
$$

При этом мы положили $E_{z} \approx 0$, что является обоснованным приближением при малых углах схождения световых пучков $2 \beta<5^{\circ}$ [3]. Используя выражения (9), можно определить состояние поляризации суммарного поля в каждой точке пространства в области перекрытия световых пучков $\mathbf{E}_{1}$ и $\mathbf{E}_{S}$. Так, выражения для параметров эллипса поляризации суммарного поля (квадратов большой и малой полуосей $a^{2}, b^{2}$, а также угла наклона $\gamma$ между большой полуосью и осью $O x$ ) в плоскости $x O y$ имеют [3] вид

$$
\begin{gathered}
a^{2}=\frac{1}{2}\left(I_{1}+I_{S}\right)+ \\
+\frac{1}{2} \sqrt{\left(I_{1}+I_{S}\right)^{2}-4 I_{1} I_{S} \sin ^{2}\left[\left(\mathbf{k}_{1}-\mathbf{k}_{S}\right) \mathbf{r}+\varphi_{1}-\varphi_{S}\right]}, \\
b^{2}=\frac{1}{2}\left(I_{1}+I_{S}\right)- \\
-\frac{1}{2} \sqrt{\left(I_{1}+I_{S}\right)^{2}-4 I_{1} I_{S} \sin ^{2}\left[\left(\mathbf{k}_{1}-\mathbf{k}_{S}\right) \mathbf{r}+\varphi_{1}-\varphi_{S}\right]}, \\
\sin 2 \gamma=\frac{2 \sqrt{I_{1} I_{S}} \cos \left[\left(\mathbf{k}_{1}-\mathbf{k}_{S}\right) \mathbf{r}+\varphi_{1}-\varphi_{S}\right]}{a^{2}-b^{2}}, \\
\cos 2 \gamma=\left(I_{1}-I_{S}\right) /\left(a^{2}-b^{2}\right) .
\end{gathered}
$$

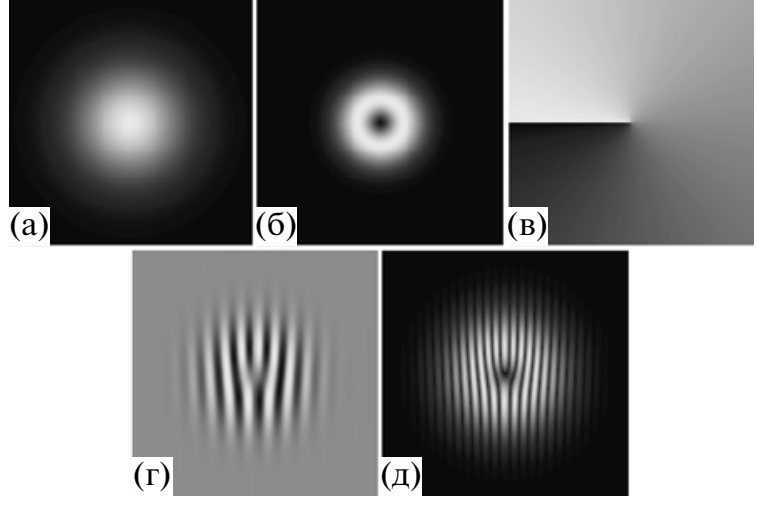

Рис. 2. Пространственные профили опорного (а) и сигнального (б) световых пучков на границе $z=0$, волновой фронт сигнального пучка (в), распределения азимута наклона (г) и отношения полуосей (д) эллипса поляризации динамической решетки.

Рассмотрим пространственные распределения параметров эллипса поляризации суммарного светового поля, приняв в качестве опорного $E_{1}$ пучок с плоским волновым фронтом и гауссовым распределением интенсивности (рис. 2a):

$$
E_{1 x}(z=0, r, \varphi)=E_{10} \exp \left[-\left(r / \sqrt{2} r_{01}\right)^{2}\right],
$$

а в качестве сигнального - вихревой пучок $E_{S} \mathrm{c}$ фазовой дислокацией топологического заряда $m$ (рис. 2б, 2в):

$$
E_{S y}(z=0, r, \varphi)=E_{S 0}\left[r / r_{0}\right]^{|m|} \exp \left[-\left(r / \sqrt{2} r_{0 S}\right)^{2}+i m \varphi\right] .
$$

Для обеспечения эффективного перекрытия пучков в объеме среды полуширина опорного пучка была выбрана в два раза большей, чем у сигнального $\left(r_{01}=2 r_{0 S}\right)$. На рис. 2г, 2д представлены рассчитанные по формулам (10)-(12) пространственные распределения азимута наклона $\gamma$ $(\pi / 2 \geq \gamma \geq-\pi / 2)$ и отношения полуосей $(\delta=$ $= \pm \operatorname{arctg}(b / a), \pi / 2 \geq \delta \geq-\pi / 2)$ эллипса поляризации суммарного светового поля на границе среды $z=0$, принимающие все возможные значения от минимума (черный цвет) до максимума (белый цвет). Видно, что в области перекрытия световых пучков формируются пространственно-периодические распределения параметров эллипса поляризации суммарного светового поля, причем решетка эллиптичности характеризуется удвоенной пространственной частотой по отношению к решетке азимута угла наклона эллипса поляризации. Наличие в поляризационных картинах вилообразных дислокаций, характерных для интерферограмм интенсивности сингулярных световых пучков [10], объясняется неопределенностью фазы в центре сигнального пучка $\mathbf{E}_{S}$. 


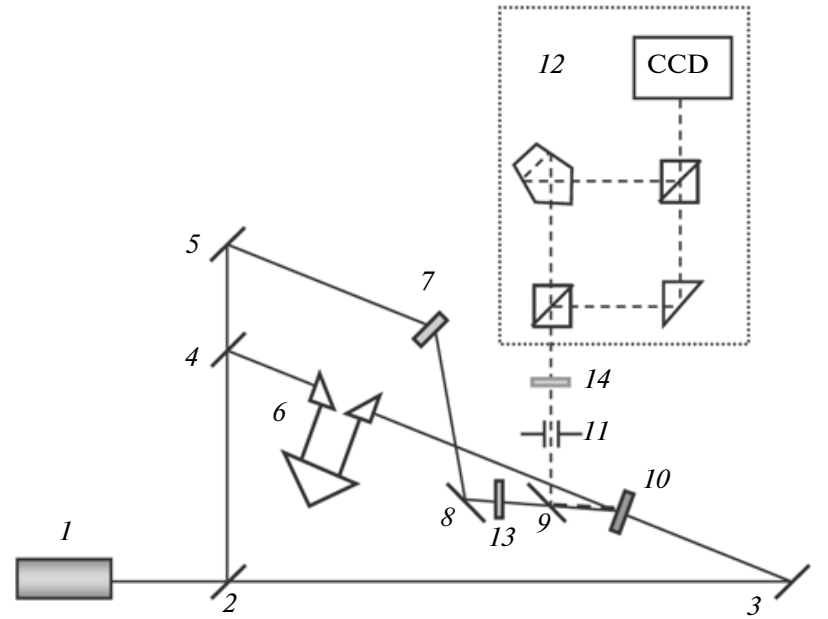

Рис. 3. Схема экспериментальной установки для реализации ЧВВ гауссовых и сингулярных световых пучков.

Таким образом, анализ структуры пространственного распределения поляризации суммарного поля опорного и сигнального световых пучков позволяет сделать вывод о том, что в средах с фотоанизотропией в рассмотренной геометрии возможна запись поляризационных голограмм, содержащих информацию о топологической структуре оптических вихрей.

При встречном направлении распространения считывающей волны

$$
\mathbf{E}_{2}=(1 / 2)\left[\mathbf{e}_{1} E_{2 x} \exp i\left(\mathbf{k}_{2} \mathbf{r}-\omega t+\varphi_{2}\right)+\text { K.c. }\right]
$$

и выполнении условия фазового синхронизма $\mathbf{k}_{D}=\mathbf{k}_{1}-\mathbf{k}_{S}+\mathbf{k}_{2}$ в среде с кубической нелинейностью индуцируется поляризация $\mathbf{P}_{\text {нл }} \sim \mathbf{e}_{2} \chi^{(3)} E_{1} E_{2} E_{S}^{*}$. $\mathrm{B}$ приближении малой дифракционной эффективности с учетом выражений (6), (7) для наведенной анизотропии уравнение для комплексной амплитуды дифрагированной волны $\mathbf{E}_{D}$ может быть записано в виде

$$
\left(\frac{\partial}{\partial z}+\beta \frac{\partial}{\partial x}+\frac{\Delta_{\perp}}{2 i k}\right) \mathbf{E}_{D}=-i \frac{k_{0}}{2} \mathbf{e}_{2} \frac{3}{8} \frac{\hat{\Theta}}{B} \alpha^{\prime} E_{1} E_{2} E_{S}^{*} .
$$

В данном уравнении $\Delta_{\perp}=\partial^{2} / \partial x^{2}+\partial^{2} / \partial y^{2}-$ поперечный лапласиан, $k=\omega n_{0} / c-$ волновое число, $k_{0}$ - линейный коэффициент поглощения среды, $\hat{\Theta}(\omega)=\Theta(\omega)+i B(\omega), \alpha^{\prime}=\alpha c n_{0} / 8 \pi$.

Как следует из анализа уравнения (13), дифрагированная волна $\mathbf{E}_{D}$ распространяется точно навстречу сигнальной волне $\mathbf{E}_{S}$ и имеет такую же поляризацию $\left(\mathbf{E}_{D} \| \mathbf{y}\right)$. При этом использование плоских опорной $\mathbf{E}_{1}$ и считывающей $\mathbf{E}_{2}$ волн $\left(\varphi_{1}+\varphi_{2}=\right.$ const $)$ позволяет реализовать эффект обращения волнового фронта, при котором фаза дифрагированной волны $\mathbf{E}_{D}$ формируется обратной к фазе сигнальной волны $\mathbf{E}_{S}\left(\varphi_{D}=-\varphi_{S}\right)$. В случае, когда сигнальный пучок $\mathbf{E}_{S}$ характеризуется наличием топологического заряда $m$, обращенный световой пучок $\mathbf{E}_{D}$ должен содержать топологический заряд обратного знака $-m$.

\section{ЭКСПЕРИМЕНТАЛЬНЫЕ РЕЗУЛЬТАТЫ}

Экспериментально ЧВВ поляризованных гауссовых и сингулярных световых пучков исследовалось при записи пропускающих динамических голограмм в схеме попутного распространения опорного и сигнального пучков в растворе красителя родамин 6Ж. Схема экспериментальной установки представлена на рис. 3. Лазер на алюмоиттриевом гранате 1 (расходимость светового пучка $\theta_{0.5} \leq 2$ мрад, длительность импульса $\tau=$ $=20 \mathrm{Hc}$ ) работал в режиме генерации второй гармоники излучения ( $\lambda=532$ нм). Опорная $\mathbf{E}_{1}$ и сигнальная $\mathbf{E}_{S}$ волны формировались светоделителем 2 и зеркалами 4, 5. Для согласования оптической длины пути сигнальной и опорной волн использовалась линия задержки 6. Для получения сигнального светового пучка с винтовой дислокацией волнового фронта использовались компьютерно синтезированные транспаранты 7, записанные в слоях полиметилметакрилата, содержащего фенантренхинон [14]. Сингулярный пучок направлялся в кювету 10 с этанольным раствором красителя родамин 6Ж под небольшим углом к опорной волне с помощью зеркала 8. Считывающая волна $\mathbf{E}_{2}$ направлялась в кювету с раствором красителя точно навстречу опорной волне $\mathbf{E}_{1} \mathbf{c}$ помощью подвижного зеркала 3. Малый угол ( $2 \beta \approx 30$ мрад) между направлениями распространения опорной и сигнальной волн и поперечные размеры пучков $\left(r_{10}=250\right.$ мкм и $r_{S 0}=75-150$ мкм$)$ обеспечивали эффективное пространственное перекрытие взаимодействующих световых пучков в кювете с раствором красителя. Для выделения дифрагированного пучка использовались полупрозрачное зеркало 9 и диафрагма 11. Пространственные профили интенсивности световых пучков регистрировались с помощью CCD-камеры, размещенной на выходе интерферометра МахаЦендера 12, позволяющего получать интерференционные картины для сигнального и дифрагированного световых пучков и идентифицировать их топологическую структуру. Поляризация взаимодействующих волн изменялась посредством внесения в сигнальный пучок полуволновой фазовой пластинки 13, а состояние поляризации обращенной волны анализировалось с помощью призмы Глана 14. При проведении эксперимента 
также отслеживался вид пространственного распределения и топологическая структура обращенной волны. Для регистрации пространственного распределения пучка одно из плеч интерферометра Маха-Цендера перекрывалось, и на камеру поступал только один пучок.

Результаты экспериментального исследования ЧВВ в этанольном растворе красителя родамин 6Ж представлены на рис. 4, где приведены изображения пучков, полученные в эксперименте (левая колонка), а также соответствующие им интерферограммы (правая колонка), подтверждающие наличие топологического заряда.

На начальном этапе все взаимодействующие волны имели одинаковую поляризацию - вертикальную, что соответствовало стандартной схеме ЧВВ. Изображения на рис. 4а', 4а" соответствуют сигнальному пучку $\mathbf{E}_{S}$. В данном случае в качестве сигнального использовался сингулярный пучок с топологическим зарядом $m=1$, что подтверждается интерферограммой, в которой, как и следует из вида используемой для регистрации заряда схемы, добавляются две интерференционные полосы. Дифрагированный пучок $\mathbf{E}_{D}$ (рис. 4б', 4б") также был вертикально поляризован и содержал топологический заряд, по модулю равный заряду сигнального пучка. Отметим, что обращение волнового фронта сингулярного пучка, реализующееся при стандартных схемах ЧВВ, приводит к инверсии знака топологического заряда [10], однако для подтверждения данного факта необходимо регистрировать интерферограммы сигнального и дифрагированного пучков не с плоским волновым фронтом, а со сферическим [12].

Путем внесения в сигнальный пучок фазовой пластинки $\lambda / 2$ была реализована схема записи поляризационной голограммы линейно поляризованными во взаимно ортогональных плоскостях опорным гауссовым и сигнальным сингулярным световыми пучками, аналогичная представленной на рис. 1. Полученные изображения дифрагированного пучка $\mathbf{E}_{D}$ и соответствующая интерферограмма представлены на рис. 4в', 4в". Поляризация дифрагированного пучка совпадала с поляризацией сигнального пучка. Помещая полуволновую фазовую пластинку поочередно в опорный и считывающий гауссовы световые пучки, были также получены и другие возможные комбинации ортогональной поляризации взаимодействующих волн. Интерферограммы, полученные при этом, показывают, что при любой комбинации поляризаций взаимодействующих волн топологический заряд, внесенный в сформированную динамическую голограмму, передается обращенной волне и по модулю равен заряду сигнальной волны $\mathbf{E}_{S}$.
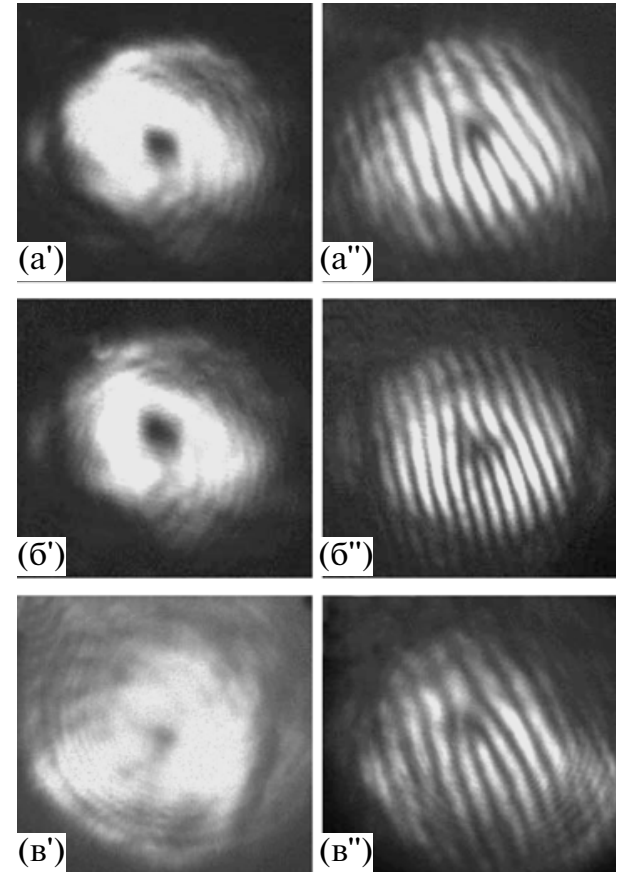

Рис. 4. Пространственные профили и интерферограммы сигнального сингулярного пучка с топологическим зарядом $m=1$ (а) и дифрагированных пучков при одинаковой (б) и ортогональной (в) поляризациях опорного и сигнального пучков.

В заключение отметим, что исследованный в настоящей работе метод записи и считывания поляризационных динамических голограмм в средах с фотоиндуцированной анизотропией коэффициента поглощения и показателя преломления открывает дополнительные возможности управления структурой сингулярных световых пучков, что может найти применение, например, при реализации математических операций с использованием в качестве логических единиц топологического заряда оптических вихрей и их состояния поляризации.

Работа частично поддержана Белорусским республиканским фондом фундаментальных исследований в рамках проекта Ф11K-136.

\section{СПИСОК ЛИТЕРАТУРЫ}

1. Какичашвили Ш.Д. // Опт. и спектр. 1972. Т. 33. В. 3. C. 324 .

2. Ebralidze T.D. // Appl. Opt. 1995. V. 34. P. 1357.

3. Huang T., Wagner K.H. // IEEE J. Quantum Electron. 1995. V. 31. P. 372.

4. Хu M., de Boer D.K.G., van Heesch C.M., Wachters A.J.H., Urbach H.P. // Opt. Expr. 2010. V. 18. № 7. P. 6703. 
5. Тарасашвили В.И., Пуриеладзе А.Л. // Опт. и спектр. 2007. T. 103. № 6. С. 1046-1049.

6. Nikolova L., Todorov T. // J. Mod. Opt. 1984. V. 31. P. 579.

7. Blouin A., Denariez-Roberge M.M. // IEEE J. Quant. Electron. 1993. V. 29. P. 227.

8. Arce Diego J.L., Fanjul Velez F., Pereda Cubian D., Tolstik A.L., Romanov O.G., Ormachea O. // Proc. SPIE. 2005. V. 5710. P. 159.

9. Карпук С.М., Рубанов А.С., Толстик А.Л. // Опт. и спектр. 1996. Т. 80. № 2. С. 313.
10. Романов О.Г., Толстик А.Л. // ЖПС. 2009. Т. 76. № 3. C. 395.

11. Романов О.Г., Толстик А.Л. // Опт. и спектр. 2008. T. 105. № 5. С. 825.

12. Романов О.Г., Горбач Д.В., Толстик А.Л. // Опт. и спектр. 2010. Т. 108. № 5. С. 812.

13. Степанов Б.И., Грибковский В.П. Введение в теорию люминесценции. Минск: Изд. АН БССР, $1963.444 \mathrm{c}$.

14. Mahilny U.V., Marmysh D.N., Tolstik A.L., Matusevich V., Kowarschik R. // J. Opt. A. 2008. V. 10. № 8. P. 085302. 